MATEC Web of Conferences 18, 01003 (2014)

DOI: 10.1051/matecconf/ 20141801003

(c) Owned by the authors, published by EDP Sciences, 2014

\title{
Some problems in nucleate flow boiling
}

\author{
C.W.M. van der Geld ${ }^{1}$, C.H.M. Baltis ${ }^{1}$, G.J.M. Priems ${ }^{1}$, T. Baki ${ }^{2}$ \\ ${ }^{1}$ Department of Mechanical Engineering, Eindhoven University of Technology \\ Den Dolech 2, 5612 AZ Eindhoven, The Netherlands \\ E-mail : c.w.m.v.d.geld@tue.nl \\ ${ }^{2}$ Faculté de Mécanique, Lab. de Carburants Gazeux et Env., Univ. S. T. Oran, M.B., Algeria
}

Keywords : Boiling, Bubble detachment, Forces on bubbles

\section{INTRODUCTION}

Although nucleate flow boiling is one of the most efficient modes of heat transfer, many of its aspects are not well understood even today. This paper intends to summarize some of important issues currently involved in measuring, interpreting and predicting nucleate flow boiling.

The topic is rich of basic physical phenomena. Understanding of all of them is required to come to reliable mechanistic models of nucleate boiling. Although such models were actively sought for (Kolev (2005) [1], Stephan and Hammer (1994) [2], Basu et al. (2005) [3]) the predictive capacity is limited and the well-known empirical correlation of Chen (1966) [4] is doing well in comparison, see Kroes et al. (2009) [5] and Fig. 1. Model predictions for cross-sectional geometries other than circular (tubes) and for channels with axially varying cross-section are awkward if the mean channel diameter exceeds $1 \mathrm{~mm}$. In the last decade, mini- and microchannels with mean channel diameter less than $0.1 \mathrm{~mm}$, were extensively studied [6] but because of the dominance of capillary forces in these channels, scaling of nucleate boiling results to larger channels is not straightforward [7].

Amongst the most difficult aspects of nucleate boiling are:

- The occurrence of a wide variety of scales

- The interplay of convection of heat and bubble motion

- The interaction of nucleation sites via convection and bubble motion.

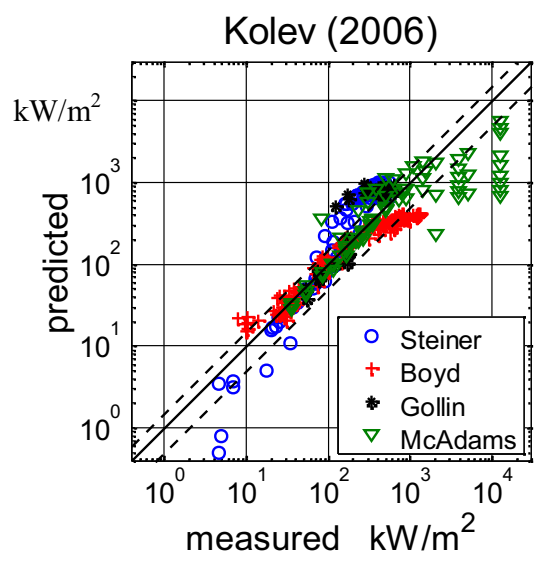

Figure 1: Validation of the Kolev model of 2006 by comparing predicted and measured heat fluxes for nucleate boiling with 4 data sets; see ref [5].

The variety in scales originates from turbulence, bubble detachment size variation and bubble coalescence, to name a few mechanisms. One of the main consequences of the occurrence of many scales is the difficulty in predicting nucleate flow boiling with full numerical solutions, because these multi-scale problems are computationally very expensive. When two bubbles coalesce, for example, the thinning of the film in between induces scales much smaller than characteristic bubble scales. Methods that solve automatically for coalescence, like the Diffuse Interface Method [8], cannot cope with realistic scales of nucleate boiling, at present.

The other difficult nucleate boiling aspects listed above have been addressed in recent studies $[9,10]$.

In the following some of the problems involved in nucleate boiling and its prediction are revisited. A survey like this can never aim to be complete, but an attempt is made to at least cover topics that have often led to misunderstanding or misinterpretation in the literature. Section 2 will start with a list of issues in the realm of bubble detachment in nucleate boiling. Section 3 presents numerical computations to illustrate some issues concerning forces on a bubble in nucleate boiling. Section 4 deals with experiments in which the orientation of the dominant body force during bubble growth, buoyancy, with respect to the heated wall is varied. Section 5 will summarize theoretical expressions for forces acting on vapor bubbles growing in a liquid flow. In addition, existing techniques to analyze bubble growth measurements are revisited in section 5 .

\section{ISSUES IN THE PREDICTION OF BUBBLE DETACH- MENT}

A criterion is often sought to determine the volume-averaged bubble diameter at detachment, $D_{d}$. Quite a few criterions exist based on empiricism and formulated in terms of the gravity constant and the surface tension coefficient, amongst other parameters. The occurrence of force components in these correlations has led people to believe that with only a force balance, one could devise a correlation to predict the bubble size at detachment. This is wrong. It is of course always possible to specify and collect force components, for example all those in the direction perpendicular to the wall, $x$. With the aid of Newton's second law, the sum of these force components can be put equal to the product of the mass of the bubble, $M_{b}$, and the $x$-acceleration of its center of mass, $d^{2} x_{C M} / d t^{2}$. The buoyancy and/or inertia forces exceed this product with $M_{b}$ to such an extent that $M_{b} d^{2} x / d t^{2}$ is negligible. The sum of all specified forces must therefore be zeor at all times. Not only at the time of detachment. Newton's law on its own does not yield a detachment size correlation.

A detachment criterion could be the specification of a certain shape at detachment in some way or the other. For example, a relation between the area of contact of the bubble content with the wall, the so-called dry contact area, $A_{\text {foot }}$, and the bubble volume can be assumed to be only valid at the time of detachment (note that $A_{\text {foot }}$ is not necessarily zero at detachment). This information can then be combined with a force balance of 
the type describe above in an attempt to derive a detachment size correlation. The first conclusion, that is sometimes not well recognized, is:

Not a force balance "per se" but a detachment criterion of a kind is needed to single out the time of detachment.

All remaining issues to be listed here concern force estimates. Force expressions are normally valid at all times and contain terms which depend on time. This is obviously true for the gravity force in the form of the product of the acceleration of gravity, $\boldsymbol{g}$, with the time-dependent bubble mass, $M_{b}(t)$. Similarly, also the added mass force contains one or more coefficients which are time-dependent. These so-called added mass coefficients depend on shape and distance to a wall, for example. The added mass force on a bubble with a foot at a wall, i.e. with a bubble with $A_{\text {foot }}$ unequal to zero, contains such coefficients. They cannot be constant, as is sometimes done, and/or based on added mass expressions of a free bubble. It will be shown in section 5 that proper expressions exist for the shape-dependent added mass coefficients of bubbles with the shape of a truncated sphere. Let $h$ denote the distance of the center of mass of the bubble to the wall. It will also be shown that the momentum balance in $x$-direction not only encompasses $d^{2} h / d t^{2}$, but also other accelerations. The second conclusion reads:

Useful expressions to compute the time-dependent added mass components exist. From these, inertia-related lift is easily computed.

Quantifications presented in section 5 will show that the added mass force contributions are usually small. Far more important are the forces related to capillarity, the so-called surface tension force, $F_{\sigma}$, and to overpressure in the bubble, the so-called pressure correction force, $F_{\Delta p}$. Let $\boldsymbol{e}_{\boldsymbol{x}}$ be the normal to the presumably flat wall, into the bubble, the unit vector in $x$-direction. The pressure correction force equals $\boldsymbol{e}_{\boldsymbol{x}}$ times $A_{\text {foot }}$ times the difference of the pressure inside the bubble, $p_{b}$, arguably homogeneous, and the pressure in the liquid around the bubble, at a height that depends on the way gravity and buoyancy are accounted for, $p_{L, f}$ :

$$
\boldsymbol{F}_{\Delta p}=\left(p_{b}-p_{L, f}\right) \boldsymbol{e}_{\boldsymbol{x}} A_{\text {foot }}
$$

In $x$-direction, the surface tension force has a contribution proportional to the surface tension coefficient, $\sigma$, and proportional to a mean value of $\sin (\theta)$, where $\theta$ denotes contact angle, measured in the fluid. Force estimates in section 5 will show that each of the magnitudes of $F_{\sigma}$ and $F_{\Delta p}$ is at least 2 orders of magnitude bigger than each of the other force component magnitudes, in $x$-direction. However, it is not difficult to derive that in case the bubble has the shape of a truncated sphere the sum of $F_{\sigma}$ and $F_{\Delta p}$ is zero. This implies that if the foot of the bubble is well approximated by the foot of a truncated sphere, that then the sum is close to zero. The area $A_{\text {foot }}$ is the area of a circle in that case. A slight deviation in bubble shape from that of a truncated sphere suffices to make the sum of $F_{\sigma}$ and $F_{\Delta p}$ at least of about the same order of magnitude as each of the other force components normal to the wall. The sum can easily balance buoyancy, for example. The shape of a bubble can be delicately tuned to compensate for forces exerted on the bubble since a minor discrepancy of the shape near the foot affects the sum $F_{\sigma}$ and $F_{\Delta p}$. Some authors introduced a fit parameter $\beta$ in the sum: $F_{\sigma}+\beta F_{\Delta p}$, in order to get agreement between measurements and predictions by fitting $\beta$. This leads to the next conclusion:

Force expressions for the capillary force, $F_{\sigma}$, and for the overpressure in the bubble, $F_{\Delta p}$, are crucial in assessing the force balance on a bubble attached to a wall.

Fit parameter $\beta$ should be 1 .

Experimental determination of these two forces, $F_{\sigma}$ and $F_{\Delta p}$, is usually done via the shape of the bubble near the bubble foot. However, particularly in the early stages of bubble growth in boiling this can be awkward because of the so-called mirage problem. The error in the contact angles measured can be significant. For this reason the following conclusion is drawn:

Expressions for the sum $\left(F_{\sigma}+F_{\Delta p}\right)$ in terms of properties of the entire bubble shape, not merely those prevailing at its foot, facilitate experimental assessment of this important force contribution.

The rise velocity of a bubble steadily rising in an unbounded liquid follows from a balance of drag and buoyancy. In common practice many other examples exist in which steady drag is important. This is not the case with vapor bubbles growing on a heated wall. Quite often in literature the drag force on such a bubble was estimated with a correlation developed for quasi-steady drag and fully developed flow over a body. This is inappropriate since a growing vapor bubble usually has a high growth rate and short lifetime, in the order of 5 to $10 \mathrm{~ms}$. In these circumstances boundary layers do not develop fully. Reasonable estimates for the time-dependency of drag and lift can be given for certain bubble shapes. Quantifications will be presented in section 5 . These are essentially overestimations that in low-viscous liquids still yield values which are negligible as compared to other force components. Drag is proportional to dynamic viscosity and its value in for example water is low. Summarizing:

Drag forces are usually negligible in vapor bubble growth in nucleate boiling.

In case a heated surface is oriented horizontally, buoyancy acts in $x$-direction, trying to free the bubble from the plate. If, additionally, the bubble shape is axisymmetric and the fluid is quiescent, only gravity and surface tension determine the bubble shape. The bubble shape can then be computed, see for example the analytical solution of Chesters [11]. These circumstances may serve as a reference for any measurement method, in particular when the contact angle is difficult to be measured. The solutions may be used to examine the force balance in $x$-direction.

Many of the statements made in the above will be detailed and explained in the following. Thereafter, measurements and analysis will be presented to substantiate the arguments given above.

\section{NUMERICAL SIMULATIONS}

In a study of the importance of various force components it makes sense to vary the importance of gravity, as this is an important body force. This can be done by reducing gravity, i.e. by performing microgravity experiments, but also by varying the angle of inclination of a boiling channel. Section 4 will present experiments of this nature. The present section deals with two-dimensional simulations which were performed in Fluent ${ }^{\mathrm{TM}}$ to help to explain force balances. Also the different trends in bubble growth and bubble detachment that occurif the angle of inclination of the test section and the flow direction are both varied will be examined. Of course, a $2 \mathrm{D}$ bubble footed at a plane wall is difficult to imagine. Despite of this and although no quantitatively directly useful results can be expected from these computations, trends are observed which in some respects are 
similar to those that will be observed in the experiment to be presented in section 4 . To facilitate interpretation, bubble volume, $V_{b}$, is taken to be constant with a constant contact angle of $54^{\circ}$. The volume equivalent radius is $1.53 \mathrm{~mm}$. The gravity constant is $9.81 \mathrm{~m} / \mathrm{s}^{2}$. The Volume Of Fluid (VOF) method is applied to compute interface deformation and motion.

Before addressing the lift force, the upstream and boundary conditions are considered. The flow is assumed to be laminar. The resulting upstream velocity profile is given by Fig. 2. A rather steep velocity increase near the wall is computed. Similarly, the temperature steeply drops from $378.15 \mathrm{~K}$ at the wall to $373.15 \mathrm{~K}$ in the bulk. The wall temperature was imposed. The volume of the bubble is constant and heat transfer does not lead to evaporation. The velocity component normal to the wall is essentially zero upstream and away from the wall and about 5 $\mathrm{mm} / \mathrm{s}$ at the height of the bubble and close to it.

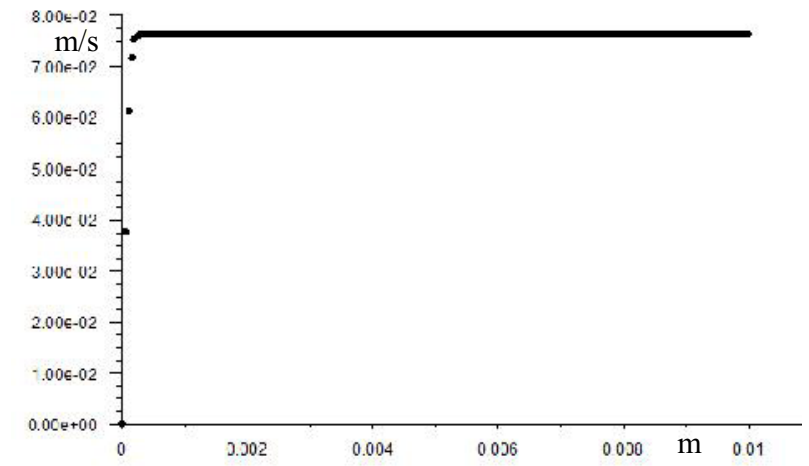

Figure 2: Velocity component parallel to the wall, in $\mathrm{m} / \mathrm{s}$, versus distance to the wall, in $\mathrm{m}$, at distance $12 \mathrm{~mm}$ upstream of the bubble center. Approximate bubble radius is $1.53 \mathrm{~mm}$.

In order to convert the findings to force balances, some additional quantities were computed. Let $A_{\text {int }}$ be defined as the area of the vapor-liquid interface. The pressure force on the bubble comprises a pressure component given by

$$
\boldsymbol{F}_{\text {lift } \boldsymbol{p}}=\iint p \boldsymbol{n} d A .
$$

where the area of integration is $A_{\text {int }}$. In the 2D computations at hand, the above surface integral reduces to a $1 \mathrm{D}$ integral over the contour, while the resulting value of $\boldsymbol{e}_{\boldsymbol{x}} \cdot \boldsymbol{F}_{l i f t, p}$ is per unit depth. So $\boldsymbol{e}_{\boldsymbol{x}} \cdot \boldsymbol{F}_{l i f t, p}$ is the component of $\boldsymbol{F}_{l i f t, p}$ in $x$-direction, $F_{l i f t, p, x}$. Pressure comprises hydrostatic and hydrodynamic parts and both occur in (2). Let the hydrodynamic part be denoted with $\boldsymbol{F}_{\text {hydrodyn }}$ and the hydrostatic part with $\boldsymbol{F}_{p}$. What is not included in (2) is the integral of the pressure inside the bubble over the foot of the bubble. This foot is defined as the dry area of the wall in direct contact with the gas content of the bubble and is denoted with $A_{\text {foot }}$. Let for vertical flows $p_{\text {stat, }}$ denote the hydrostatic head in the liquid at the height of the center of mass of the bubble be. Let for horizontal flows $p_{\text {stat }, c}$ denote the hydrostatic head in the liquid at the wall. Angles of inclination other than the vertical and horizontal ones are not considered in this paper. The sum

$$
\boldsymbol{F}_{p}+p_{\text {stat }, c} A_{\text {foot }} \boldsymbol{e}_{x}
$$

is equal to the buoyancy force on the bubble, $\boldsymbol{F}_{\text {buo }}$. Fluent computations directly provide the pressure inside the bubble, $p_{b}$, whereas $p_{\text {stat },}$ in the vertical case requires determination of the center of mass. The magnitude of the force on the foot,

$$
F_{\text {foot }}=p_{b} A_{\text {foot }}
$$

is therefore more readily determined. Hydrodynamic stresses also encompass viscous stresses. The last one was found to be at least three orders of magnitude less than the pressure part, $F_{l i f t, p, x}$, and will therefore not be considered further.

The overpressure in the bubble, $\Delta p$, is defined as the

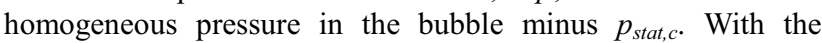
neglect of viscous stresses, the dynamic boundary condition yields

$$
p_{b}=\Delta p+p_{\text {stat }, c} .
$$

In the Fluent computations, the value of $\Delta p$ is 10 to $20 \mathrm{~Pa}$, while the value of $\sigma$, the surface tension coefficient, is $0.0589 \mathrm{~N} / \mathrm{m}$. Note that curvature contains only one radius of curvature because of the 2D situation.

In vertical upflow, the bubble is found to move upward and drag fluid with it. As a consequence, velocity near the wall is higher than at corresponding locations in the approaching velocity field of Fig. 2, while further away from the wall it is lower. Pressure $p_{\text {statc }}$ decreases in the course of time, so the pressure in the bubble decreases as well, see Fig. 3. The product of the bubble volume and the pressure in the bubble is not constant but the bubble volume, $V_{b}$, is.

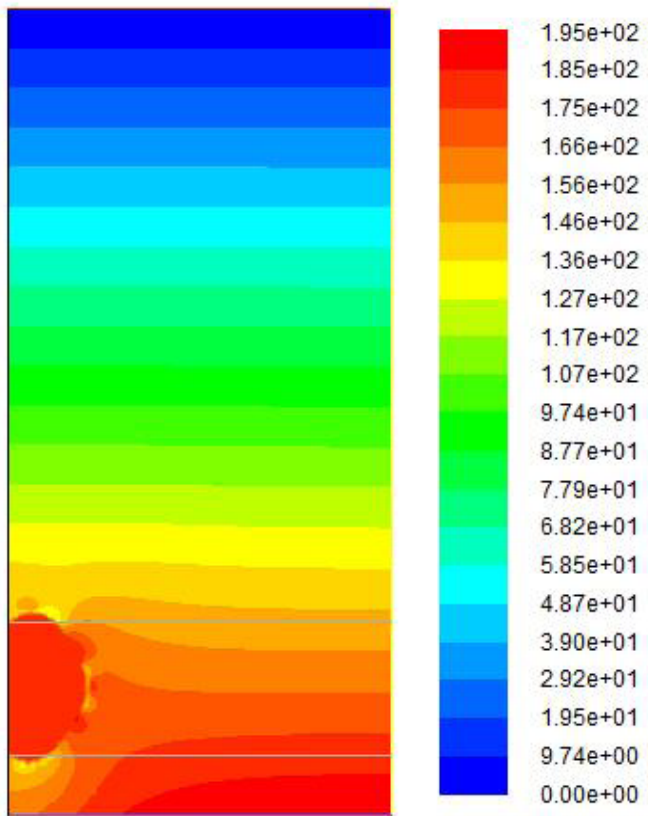

Figure 3: Static pressure field around a 2D bubble rising vertically along a plate. Values are in Pa; gravity is pointing downward; liquid flow is upward. The width of the figure in horizontal direction measures $20 \mathrm{~mm}$; approximate bubble radius is $1.53 \mathrm{~mm}$.

The pressure correction force is defined by (1) and can be written as

$$
\boldsymbol{F}_{\Delta p}=A_{\text {foot }} \Delta p \boldsymbol{e}_{x} .
$$

The order of magnitude per unit depth is 0.002 times $20 \mathrm{~Pa}$, or $0.04 \mathrm{~N}$. Its value is decreasing in time since $A_{\text {foot }}$ is found to be decreasing over time and because the overpressure is not varying substantially. The capillary force per unit depth,

$$
\boldsymbol{F}_{\sigma}=-2 \sin \left(\theta_{c}\right) \sigma \boldsymbol{e}_{x},
$$

with $\theta_{c}$ the constant contact angle and the factor 2 accounting for the two sides, has the same order of magnitude as the pressure correction force. However, the sum $\left(F_{\Delta p}+F_{\sigma}\right)$ is small since these two forces are counteracting: capillary is attracting to the 
wall while overpressure pushes the bubble away from the wall. The sum of their $x$-components is precisely counteracting the sum of buoyancy and hydrodynamic forces, $F_{\text {lift,p,x }}+p_{\text {stat }, c} A_{\text {foot }}$ :

$$
F_{\Delta p}+F_{\sigma}+F_{\text {lift, }, x}+p_{\text {stat }, c} A_{\text {foot }}=0 .
$$

The smaller the bubble foot, the smaller the asymmetry of the liquid-vapor interface and the smaller the magnitude of the lift force $F_{l i f t, p, x}$ therefore is. This lift component starts of as a relatively strong negative force component. Negative means towards the wall, clearly a consequence of the pressures on top of the bubble. Since $F_{\sigma}$ is about constant and since both $F_{\Delta p}$ and $p_{\text {stat }, c} A_{\text {foot }}$ are decreasing in time in the vertical case of Fig. 3 , the increasing $F_{l i f t, p, x}$ compensates the other force components.

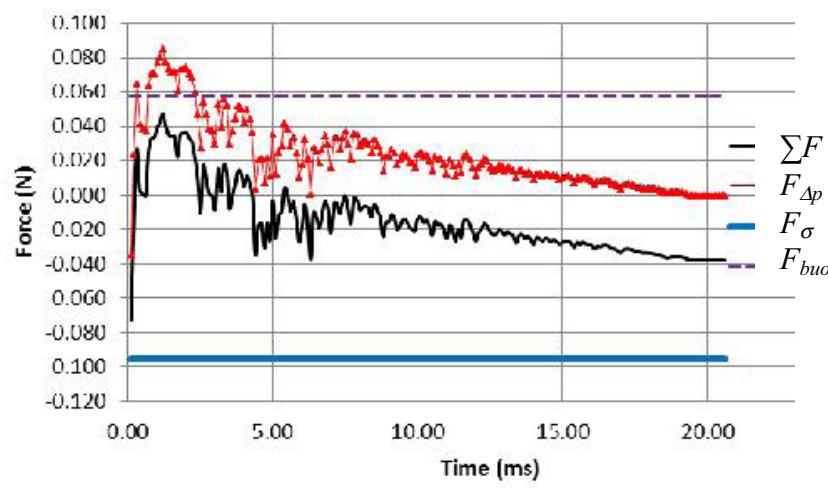

Figure 4: Forces histories for normal horizontal flow, case $I$. The inaccuracy of the overpressure, $\Delta p$, and the unknown hydrodynamic contribution, $F_{\text {hydrodyn, make the }}$ sum of the components given, $\sum F$, unequal to zero.

Although the alternative form of (8),

$$
F_{\sigma}+F_{l i f t, p, x}+F_{\text {foot }}=0
$$

is more readily assessed from numerical computations, some of its terms depend on the hydrostatic pressure. The alternative form

$$
F_{\Delta p}+F_{\sigma}+F_{\text {buo }}+F_{\text {hydrodyn }}=0
$$

merely contains terms that do not necessarily depend on the height of the bubble center. The buoyancy force, $\boldsymbol{F}_{\text {buo }}$, equals the product $-V_{b} \boldsymbol{g}\left(\rho_{L}-\rho_{V}\right)$ while the $\mathrm{x}$-component of the hydrodynamic force, $F_{\text {hydrodyn }}$, is the part of $F_{\text {lift,p,x }}$ that is not related to hydrostatic pressure. Figure 4 evaluates (10) for the case of horizontal flow when the bubble eventually leaves the plate. As expected, $F_{\Delta p}$ goes to zero at the time of detachment because then the area $A_{\text {foot }}$ is computed to go to zero. Since in this simplified computation both the buoyancy force and the capillary force are constant, the force $F_{\text {hydrodyn }}$ not shown in Fig. 4 , must account for the changes in $F_{\Delta p}$. The verification of this with the aid of (9) is work in progress.
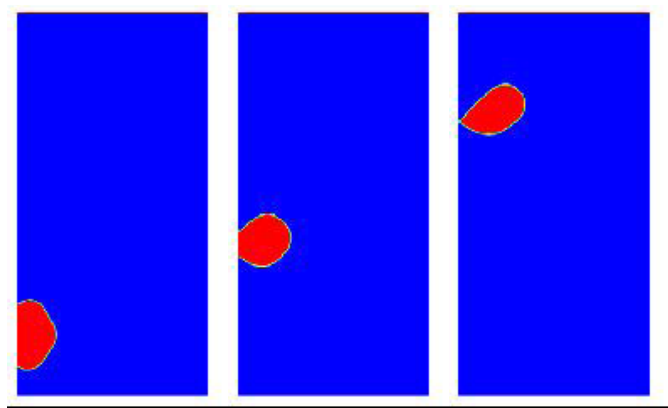

$$
t=0.003 \mathrm{~s} \quad \mathrm{t}=0.03925 \mathrm{~s} \quad \mathrm{t}=0.0785 \mathrm{~s}
$$

Figure 5: Shape history of a 2D bubble rising vertically along a plate. Times: $0.003 \mathrm{~s}$ (left), $0.03925 \mathrm{~s}$ (middle), 0.0785 $s$ (right). Gravity is pointing downward; liquid flow is upward. The width of each figure in horizontal direction measures $20 \mathrm{~mm}$; approximate mean bubble radius is 1.53 mm.

In the standard upward flow case, the bubble shape just before detachment is inclined upward, in the same direction as the flow, as if the bubble top is moved upward with respect to the bubble foot, by buoyancy or flow pressure, see Fig. 5 (right).

The lift force part $F_{\text {lift, }, x}$ turns out to be positive in the vertical downflow case, while the bubble leaves its original axial $y$-position only a little before detachment in the downflow case computed (Fig. 6, left). As a consequence, the $x$-component of the velocity is essentially zero in the liquid phase, while its $y$-component parallel to the wall is slightly increased next to the bubble because of mass conservation. The hydrodynamic pressure decrease on the side of the bubble that is not facing the wall apparently compensates the gravitational head. This pressure reduction does not occur in upward flow since the bubble is sliding along the wall in this case.

In this downward case, the bubble is inclining upward, against the flow, whose direction in Fig. 6 is from bottom to top. This leads us to believe that in these 2D computations the bubble shape is merely determined by buoancy.

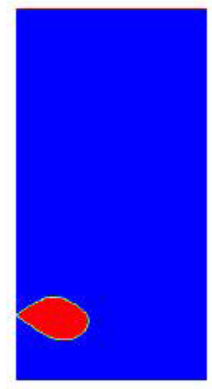

Case III

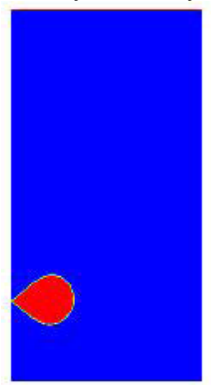

Case I

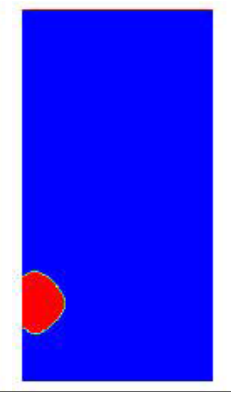

Case II
Figure 6: Three final shapes after $0.0665 \mathrm{~s}$ in downflow (left), after $0.0206 \mathrm{~s}$ in horizontal flow with gravity towards the wall (middle, bubble detaches) and after $0.0206 \mathrm{~s}$ in horizontal flow with gravity away from the wall (right). Liquid flow is from the bottom of the figure to the top, in all three cases. The width of each figure in horizontal direction measures $20 \mathrm{~mm}$; approximate mean $2 \mathrm{D}$-bubble radius is $1.53 \mathrm{~mm}$.

\begin{tabular}{|l|l|l|l|}
\hline Case \# & $g_{x}$ & $g_{y}$ & flow \\
\hline I & -9.81 & 0 & horizontal \\
\hline II & 9.81 & 0 & horizontal \\
\hline III & 0 & 9.81 & downflow \\
\hline IV & 0 & -9.81 & upflow \\
\hline
\end{tabular}

Table 1: Four generic case definitions. In the numerical computations, uy is always positive.

In the normal horizontal case (Case I; final shape in Fig. 6, middle), the lift part $F_{\text {lift, }, x}$ is negative and the bubble does not move axially. In the upside-down horizontal case (Case II; Fig. 6, right), $F_{l i f t, p, x}$ is positive and the bubble cannot escape its original position, not in horizontal axial $y$-direction nor in the $x$-direction normal to and away from the wall.

These cases I and II are defined in Table 1, that also defines the other two cases. This case numbering will also be used in section 4. In Case III, the flow is vertical and parallel to $g$, i.e. in downflow. A positive lift $F_{l i f t, p, x}$ is computed in this case III. In 
the horizontal upside-down case II, hydrostatic pressure under the bubble is higher than the hydrostatic pressure at the wall. This causes a lift contribution towards the wall, i.e. negative. Also in the normal horizontal case I and the standard vertical upflow case (Case IV) the lift $F_{l i f t, p, x}$ is found to be negative. The predicted $2 \mathrm{D}$ bubble deformations and bubble motions will be compared with new, 3D experiments in the following section, 4, for all four cases of Table 1. It will be attempted to interpret trends found with the aid of the above computational results of $F_{\text {lift, }, x}$.

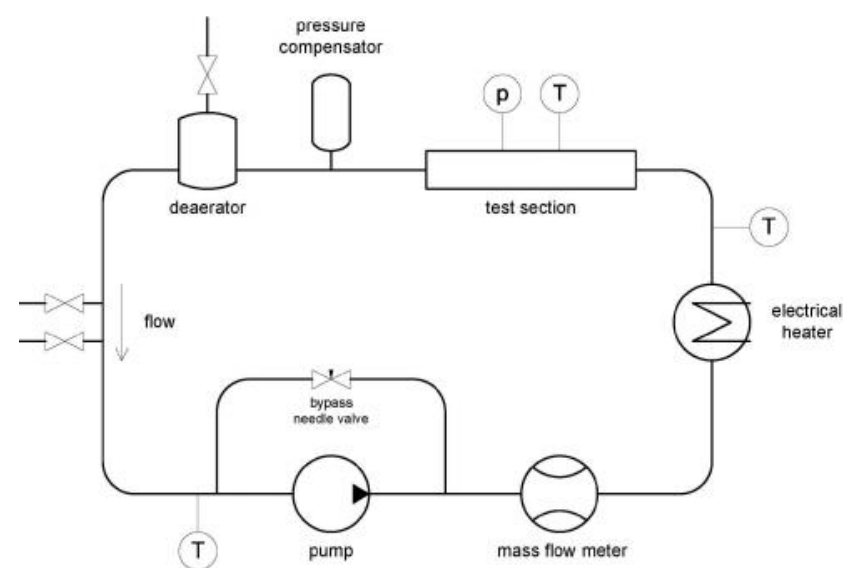

Figure 7: Schematic of the experimental setup. The bubble generator is situated in the test section.

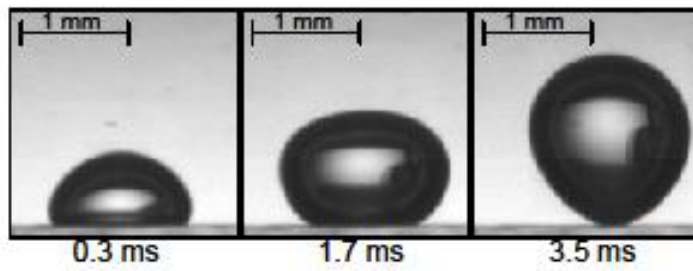

Case I

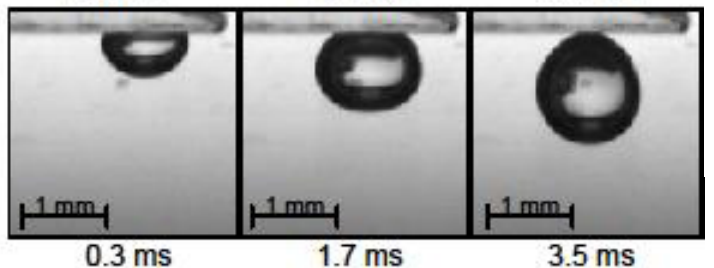

Case II

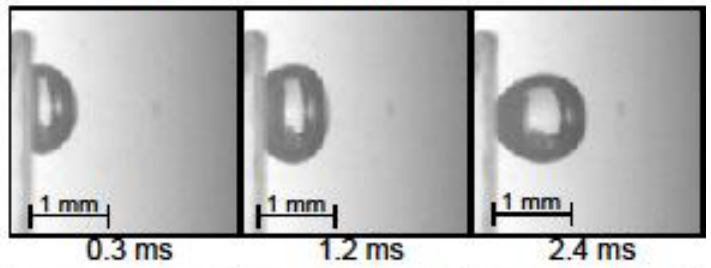

Case III

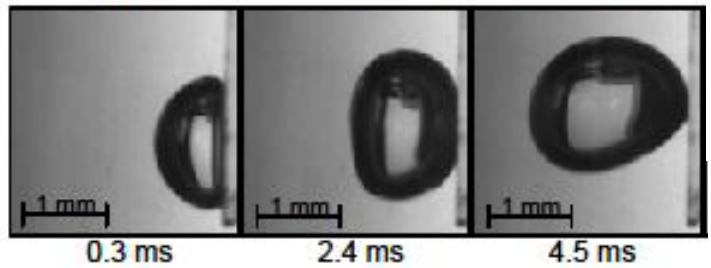

Figure 8: Bubble growth and detachment in the four

Table 1. The arrows denote gravity and bulk velocity final times given are just prior to bubble detachm

\section{EXPERIMENTS}

\subsection{Test rig and measurement strategy}

In order to investigate single vapor bubble growth and detachment in the four cases defined in Table 1, a dedicated experimental setup was designed. It facilitates optical measurement of bubble shapes and bubble motion in a test section from various sides simultaneously. In a channel with a rectangular cross-section of $5 \times 40 \mathrm{~mm}^{2}$ water is pumped around at regulated pressure, see Fig. 7. Temperature, pressure and mass flow of the water flow are measured and controlled to preset values. The approaching water temperature is just below saturation value. A bubble generator is a piece of glass with a plane side, on which a heated area of about $1 \times 1 \mathrm{~mm}^{2}$ is situated. This heated area generates single, isolated boiling bubbles. The generator is positioned in the center of the rectangular channel in order to obtain a uniform approaching flow of demineralized, degassed water at the front end of the bubble generator. The heated area starts $0.5 \mathrm{~mm}$ from the sharp front end of the glass bubble generator. The temperature of the heated surface of the bubble generator and the power fed to the bubble generator are measured. The velocity of the flow approaching the bubble generator in the center of the channel is $0.086 \mathrm{~m} / \mathrm{s}$. The bulk velocity is somewhat less and is fairly close to the bulk velocity used in the computations of section 3 .

With the aid of a high speed video camera, a high power LED array, and a mirror, the side view and the top view of growing vapor bubbles are captured. Figure 8 presents the main side views measured.

The contact line and bubble shape in the region close to the wall are important not only because the heat transfer rate near the contact line is high, but also because they determine the adhering capillary force, see (7). Bubble detachment and closing of the bubble contour, i.e. disappearing of the contact line, can experimentally be observed from ripples and other bubble shape deformations occurring at detachment. In addition, the high-speed video recordings of the bubble from above, i.e. at an angle perpendicular to the wall, can be used to visualize the contact line and the bubble foot [10]. Each of the captured side images has been digitized and analyzed. Histories of characteristic lengths scales are produced (Fig. 9). From these histories, the forces acting on the bubble are assessed in the way described in section 5. Figure 9 shows that the new measurements possess a remarkably high reproducibility.

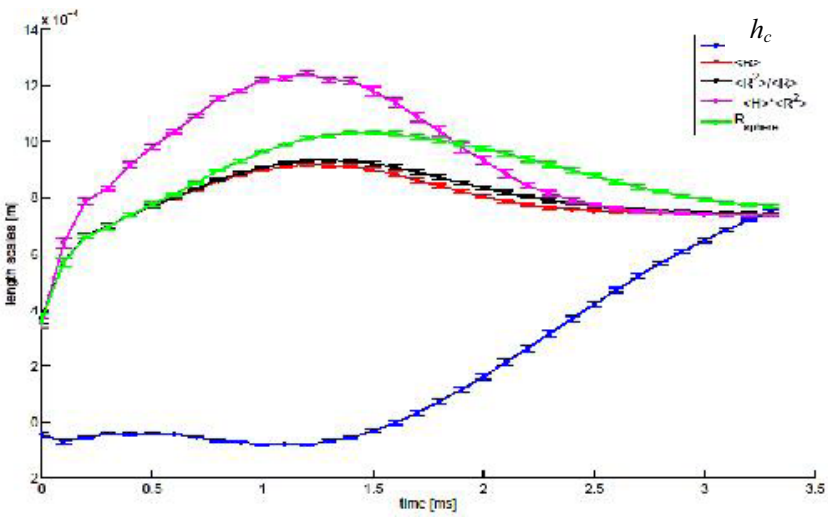

Figure 9: Histories of distance of the center point to the wall, $h_{c}$, of the mean radius, $<R>$, of the mean curvature scaled with the mean square radius, $-<H><R^{2}>$, and of the radius of a best fitting sphere, $\boldsymbol{R}_{\text {sphere }}$. The bars show the total variation of these length scales for 15 bubbles originating from the same site in the same flow situation

\subsection{Deformation history analysis}

Figure 8 summarizes the main trends observed in growth and 
in motion of the deforming bubbles. In the upflow case IV, the bubble is found to move upward. More precisely, the contact line on the upstream side is found to move towards the position of the contact line at the downstream side. A similar behavior has been found in a recent study [10]. The shape is somewhat pending against gravity, which differs from the situation in Fig. 5. This difference is probably a consequence of the uniform velocity profile, since in the study [10] a similar bubble generator was mounted flush in the wall and yielded final shapes closer to those of Fig. 5. As a matter of fact, bubbles were found to deform strongly into flow direction while the upstream side rose nearly perpendicular to the contact line and the wall. Such a situation does not develop in the present experiments because of the higher water velocities in the vicinity of the plate.

The axi-symmetry is preserved in the shapes of the two horizontal cases I and II (Fig. 8), as they were in the computations (Fig. 6). In the downflow case III, a downward motion is observed and a nearly symmetrical shape at all times. The shape differs from the computations (Fig. 6). The growth time in the downflow case III is experimentally observed to be considerably shorter than that in the upflow case IV (Fig. 8). This is interpreted as a consequence of lift, since a positive lift $F_{l i f t, p, x}$ is computed in this case III, whereas a negative lift $F_{\text {lift,p, } x}$ is computed in all three other cases. It is noted that this lift comprises both hydrostatic and hydrodynamic components. The interpretation of the first part is given by Eq. (3). Prediction and interpretation of hydrodynamic lift is dealt with in section 5 .

\section{FORCE ANALYSIS}

\subsection{Capillarity and other dominant forces}

The force components acting on a growing bubble normal and parallel to the wall are of course important for the prediction of the sliding along and detachment from the wall. Motion normal to the wall is described by the history of the distance of the center of mass of the bubble to the wall, $h$. It is noted that the center distance $h_{c}$ of Fig. 9 differs from $h$. The central point of the best fitting sphere, with radius $R_{\text {sphere }}$, has a distance $h_{c}$ to the wall; details of such a fit are given in [12]. In the force balances of this section, only $h$ is employed. Other length scales occurring in Fig. 9, like $<R>$, will be explained below (13). Another distance is connected to the sliding motion along the wall, in $y$-direction, (Fig.'s 5 and 8). To keep the discussion focused to actual detachment from the wall, motion in $y$-direction is not considered here. The most essential parameters and forces needed to describe growth and deformation of a vapor bubble attached to a plane wall will be investigated in this section.

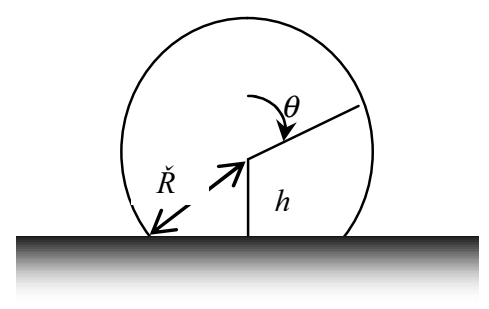

Figure 10: Definition of coordinate and length scales.

In the literature, the bubble shape is quite often described by $h_{c}$ and the radius $R_{\text {sphere }}$ only. For small bubbles this approximation is appropriate, but with fluid flow, gravity or at low system pressure, quite some deformation is possible. With arbitrary deformation, more parameters than $h$ are necessary to describe growth and detachment. In [11], a representation with Legendre polynomials is used. Here, a similar representation, but with the center point at distance $h$ from the wall, is used. It is not straightforward to make a connection with the appealing generalized coordinate system of $\left(R_{\text {sphere }}, h_{c}\right)$ and other coordinate systems when the bubble shape is not that of a truncated sphere. The problem is the arbitrariness of the definition of the sphere that must yield both $R_{\text {sphere }}$ and $h_{\mathrm{c}}$. The sphere must be close to the actual, non-spherical shape, but the only unique way to define such a sphere is to use the mean curvature of the top of the bubble, the point that is farthest away from the wall. This not always yields satisfactory fits of the vapor-liquid interface.

Let $U$ be the time rate of change of $h$ and $V$ the approach velocity parallel to the wall of the fluid at the center, at distance $h$ from the wall; $t$ denotes time. A force balance, will be derived in terms of $d U / d t$ and other accelerations, which will now be defined. With $d U / d t$, the motion of the bubble normal to the wall is easily predicted.

Define $z=\cos (\theta)$ with $\theta$ the polar angle measured from the top of the bubble in the polar coordinate system centered at center; see Fig. 10. Let $\theta_{l}$ be the angle corresponding to the foot of the bubble at the chosen wall, corresponding to radial distance $\check{R}$ of this foot, such that $\cos \left(\theta_{l}\right)=-h / \check{R}$. Only if for all $\theta$ the radial distance $R\left(\cos (\theta)\right.$ of the contour equals $R_{\text {sphere }}$ the shape is that of a truncated sphere. The function

\section{$R(\cos (\theta), t)$}

represents the measured contour with the first coordinate in the domain $\left[\cos \left(\theta_{I}\right), 1\right]$. This domain must be extended to $[-1,1]$ in such a way that the bubble foot and the contact angle are conserved and that the coefficient $b_{2}$ in the expansion

$$
R=\sum_{\mathrm{j}} b_{j} \operatorname{Le}_{\mathrm{j}-1}(\cos (\theta))=\sum_{\mathrm{j}} b_{j} \operatorname{Le}_{\mathrm{j}-1}(z)
$$

is zero. The first requirement regarding the shape requires a smooth extrapolation with first and second order derivatives of $R$ with respect to $z$ preserved. The second requirement stems from the fact that $b_{2}$ describes motion of the bubble as a whole and puts a constraint on the expansion coefficients, $\left\{b_{j}\right\}$. It is now assumed that this is successfully done and that $h$ replaces $b_{2}$. From now on, $b_{2}=h$. It is noted that when deformation in $y$-direction occurs, (11) must be extended with an extra coordinate, the azimuthal angle, and with other elementary functions. For sake of clarity only the axisymmetric case described by (11) is considered here. Let

$$
\text { ю }=\cos \left(\theta_{l}\right) \text {. }
$$

Note that $ю=\cos \left(\theta_{l}\right)=-h / \check{R}$. Brackets are used to indicate an average over the entire gas-liquid interface. For example, the average $\langle R>$ is given by

$$
\begin{aligned}
<R>= & \text { ю } \int^{1} d z R(z) /(1-\text { ю })= \\
& \left\{\int_{0} \int^{\theta 1} d \theta R(\theta) \sin (\theta)\right\} /\left(1-\cos \left(\theta_{l}\right)\right) .
\end{aligned}
$$

These brackets occur in expressions that can be derived for each of the forces occurring in the following force balance. Positive integer $j$ numbers the coordinates. The more coordinates, the higher the accuracy is:

$$
-F_{\mathrm{j}}^{\text {inertia }}-F_{\mathrm{j}}^{\text {vort }}=F_{\mathrm{j}}^{\Delta \mathrm{p}}+F_{\mathrm{j}}{ }^{\sigma}+F_{\mathrm{j}}^{\mathrm{g}}+F_{\mathrm{j}}^{\text {drag }}
$$

Force $F_{\mathrm{j}}^{\text {vort }}$ accounts for hydrodynamic forces that cannot be described with a velocity potential, $F_{\mathrm{j}}^{\text {inertia }}$ for those that depend on a velocity potential only. The lift of $F_{\mathrm{j}}^{\text {inertia }}$ and $F_{\mathrm{j}}^{\text {vort }}$, as well as the drag of $F_{\mathrm{j}}^{\mathrm{drag}}$ will be considered in section 5.2. Although the forces on the RHS of Eq. (14) look familiar for the case $j=2$, each $F_{j}$ represents a generalized force. The derivation of 
expressions for these forces is not straightforward [12]; some important examples are given below. Each of the $b_{j}$ is considered as a generalized coordinate and the corresponding Euler-Lagrange equations are put in the form (14).

The pressure difference between the hydrostatic pressure at a point at the wall and the homogeneous pressure inside the bubble, $\Delta p$, is for each generalized coordinate the same. The corresponding generalized force is given by

$$
F_{j}^{\Delta p}=\Delta p \partial V_{b} / \partial b_{j}
$$

In the vertical cases III and IV, see Table 1, the pressure drop $\Delta p$ is connected to the gravity and buoyancy force, collected in $F_{j}^{g}$, because of the choice of the reference point at the wall. The interface average $<R^{2}>$ is used to assess $\partial V_{b} / \partial b_{l}$ :

$$
\partial V_{b} / \partial b_{I}=2 \pi(1-ю)<R^{2}>
$$

The gravitational generalized force is for $j=2$ familiar:

$$
F_{2}{ }^{\mathrm{g}}=g \rho_{L} V_{b}
$$

The volume of the bubble, $V_{b}$, is given by

$$
V_{b}=2 / 3 \pi(1-ю)<R^{3}>+1 / 3 \pi h\left(\check{R}^{2}-h^{2}\right)
$$

Other expressions for generalized forces are more complicated. The one of the capillary force $F_{2}^{\sigma}$, for example, contains the curvature at each point of the liquid-gas interface and is given by

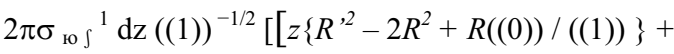

$$
\begin{aligned}
& \left.\left\{R^{\prime}((0)) /((1))-2 R R^{\prime}\right\} \sqrt{ }\left(1-z^{2}\right)+R R^{\prime} z^{2} / \sqrt{ }\left(1-z^{2}\right)\right]
\end{aligned}
$$

with $((0))=\left(R^{2} R^{\prime \prime}-R R^{, 2}\right)$ and $((1))=R^{2}+R^{\prime 2}$ with $R^{\prime}=\partial R / \partial \theta$. It can be proven that the expression (17) reduces to

$$
-2 \pi R_{\text {foot }} \sigma \sin \left(\theta_{c}\right)
$$

with $\theta_{c}$ the contact angle at the bubble foot measured in the liquid. Although this is a familiar expression, again, the accuracy of the determination of the contact angle often leaves much to be desired, see also Fig. 8. In this case a surface integral like (17) might yield higher accuracies. Moreover, the sum of two dominant forces, $F_{\mathrm{j}}^{\Delta \mathrm{p}}+F_{\mathrm{j}}{ }^{\sigma}$, can also be determined as a surface integral [12], namely the average difference of the main radii of curvature, $\left\langle 1 / R_{2}-1 / R_{1}>\right.$. This is important because these two dominating forces are compensating one another to a large extent, making the sum substantially smaller than each of the forces on its own; see section 3. Both $F_{\sigma}$ and $F_{\Delta p}$ are orders of magnitude bigger than each of the other forces acting on a bubble [12]. By tweaking the shape, the bubble adjusts the balance of the two dominating forces and makes Newton's second law satisfied at all times. Direct determination of the sum $\left(F_{\mathrm{j}}^{\Delta \mathrm{p}}+F_{\mathrm{j}}{ }^{\sigma}\right)$ from a single measurable quantity, $\left\langle 1 / R_{2}-1 / R_{1}\right\rangle$, is more accurate than summing two measured quantities.

However, in the derivation of the expression for $\left(F_{\mathrm{j}}^{\Delta \mathrm{p}}+F_{\mathrm{j}}^{\sigma}\right)$ in terms of the mean difference of the radii of curvature, the hydrodynamic pressure was neglected. Since in section 4.2 it was concluded that hydrodynamic contributions may explain differences in growth and detachment trends observed, a way to account for the hydrodynamic contribution is presently being sought. This is work in progress.

The Euler-Lagrange equation corresponding to $b_{l}$ is the Rayleigh-Plesset equation for a bubble growing at deforming at a plane wall. The Rayleigh-Plesset equation is sometimes thought to be available in a version that applies to detached bubbles only. This is of course not the case.

One of the advantage of the Euler-Lagrange equations (14) is the occurrence of the same unknown pressure difference between the inside of the bubble and the pressure at the wall, $\Delta p$, in each of them. The more coordinates can be determined from experiments, the more independent equations are obtained for the same unknown $\Delta p$.

The pressure drop $\Delta p$ is related to the heat transfer to the bubble. Prediction of its value requires the solution of the heat transfer problem, a Stefan kind of problem in which the velocity of the interface is nearly equal to that of the liquid. The temperature at a distance from the bubble is preferably known and prescribed. If so, heat transfer to the bubble can in principle be computed, although the solution is not trivial.

\subsection{Drag and lift}

Let $d R_{0} / d t$ be defined as the time rate of change of $b_{1}$ at initial time zero, $d h_{0} / d t$ that of $h$ at time zero, and let $R_{0}$ be the initial value of $b_{1}$. In boiling, $d R_{0} / d t$ is nonzero and positive which makes it a good reference velocity. The velocities $d R_{0} / d t$ and $d h_{0}$ ldt are coupled since $h$ and $b_{1}$ are coupled by the governing equations. The velocity of the approaching flow at the center of the bubble, $V$, is an independent velocity. Three Reynolds numbers exist:

$$
\begin{aligned}
& \operatorname{Re}_{1}=R_{0} d R_{0} / d t \rho_{L} / \mu, \\
& \operatorname{Re}_{2}=R_{0} d h_{0} / d t \rho_{L} / \mu, \\
& \operatorname{Re}_{\mathrm{V}}=V R_{0} \rho_{L} / \mu .
\end{aligned}
$$

The occurrence of three Reynolds numbers already indicates that standard correlations for steady drag are of little use. There is time needed for boundary layers to develop. During fast bubble growth, $R e_{1}$ is significant and insufficient time is available for boundary layer development. In the following, approximate expressions will be given for the drag and lift on a hemispherical bubble growing on a plane wall.

The following functions of time, $f_{l}$ and $f_{2}$, were shown [13] to be important to account for boundary layer development on a growing sphere $\left(v=\mu / \rho_{L} ; \operatorname{Re}_{\mathrm{V}}>1\right)$. They are needed in the following:

$$
\begin{aligned}
f_{l}(t)= & \exp \left(9 v_{0} \int^{t} R\left(t^{\prime}\right)^{-2} d t^{\prime}\right) \operatorname{erfc}\left(\left\{9 v_{0} \int^{\mathrm{t}} R\left(t^{\prime}\right)^{-2} d t^{\prime}\right\}^{0.5}\right) \\
f_{2}(t)= & (1 / R(t))_{0} \int^{\mathrm{t}} \exp \left(9 v_{\tau} \int^{\mathrm{t}} R\left(t^{\prime}\right)^{-2} d t^{\prime}\right) . \\
& \operatorname{erfc}\left(\left\{9 v_{\tau}{ }^{\mathrm{t}} R\left(t^{\prime}\right)^{-2} d t^{\prime}\right\}^{0.5}\right) \partial R(\tau) / \partial \tau d \tau
\end{aligned}
$$

For a growing bubble with the shape of a hemisphere the timescales of bubble expansion and diffusion of linear momentum experience the same competition. We therefore postulate that the time dependent drag is in this case approximately represented by

$$
\begin{aligned}
&-F_{D}= 12 \pi \mu R(t) V+5 \pi \mu V\left(1-\tanh \left(\operatorname{Re}_{\mathrm{V}} / 70\right)\right) . \\
&\left(-R(t)+R_{0} f_{l}(t)+R(t) f_{2}(t)\right),
\end{aligned}
$$

where $f_{l}$ and $f_{2}$ are in principle different from those given above but possess the same asymptotic behavior. For lack of alternatives, (20) is used in combination with (21). For truncated spheres, the first two Euler-Lagrange equations, corresponding to $b_{1}$ and $h=b_{2}$, each have their own drag. Expressions are given in [12].

In analogy to the drag force treatment above, the lift on a hemisphere with radius $R(t)$ is in first order approximation given by 


$$
\begin{aligned}
& F_{2}^{l i f t}=1 / 4(11 / 8) \pi \rho R(t)^{2} V^{2}+1 / 4(11 / 8) \pi \rho R(t)^{2} V^{2} . \\
& \left(\left(0.3+0.0232 \operatorname{Re}_{\mathrm{V}}^{0.5}\right) /\left(1+0.02 \operatorname{Re}_{\mathrm{V}}\right)\right) . \\
& \left(-1+\left(R_{0} / R(t)\right) f_{l}(t)+f_{2}(t)\right) .
\end{aligned}
$$

For an expanding bubble with the shape of a truncated sphere this lift is similar, but with other constants and with the derivative $\partial \alpha_{2} / \partial h$ in each term. An explicit expression for the added mass coefficient $\alpha_{2}$ is given in the appendix of [12].

Numerical assessment of the expressions for drag and lift for truncated spheres showed that drag is negligible and that lift is well approximated by the potential flow solution [12].

\section{CONCLUSION}

This paper presented new measurements of vapor bubble growth and detachment for characteristic flow cases (Table 1). Trends observed have been interpreted and compared with 2D numerical simulations. Since most analyzing methods concern force balances, expressions for the forces acting on a growing vapor bubble attached to a plane wall have been revisited. Some misunderstandings in the literature have been examined with the aid of the force expressions and balances presented.

The combination of dedicated experiments and physical modeling presented in this paper may serve to facilitate analysis of future experiments in nucleate boiling.

\section{Acknowledgement}

This research is supported by the Dutch Technology Foundation STW, which is part of the Netherlands Organisation for Scientifc Research (NWO), and which is partly funded by the Ministry of Economic Affairs. Furthermore, this work is conducted under the umbrella of the COST MP1106 Action: Smart and Green Interfaces - from single bubbles and drops to industrial, environmental, and biomedical applications.

\section{REFERENCES}

1. N.I. Kolev, How accurately can we predict nucleate boiling? Exp. Thermal and Fluid Science, Vol 10 pp 370-378, 1995.

2. P. Stephan and J. Hammer, "A new model for nucleate boiling heat transfer," Wärme- und Stoffübertragung, 30, pp. 119-125, 1994.

3. M. Basu, G.R. Warrier and V.K. Dhir, Wall heat flux partitioning during subcooled flow boiling: Part 1 - model development, J. Heat Transfer, 127, pp. 131-140, 2005.

4. J.C. Chen, Correlation for boiling heat transfer to saturated fluids in convective flow, Ind. \& Eng. Chem., 5(3), pp. 322-329, 1966.

5. J.P. Kroes, C.W.M. van der Geld and E. van Velthooven, Evaluation of four nucleate flow boiling models, Advances in Multiphase Flow and Heat Transfer, 1, pp. 267-283, 2009.

6. C. Rops, G. Oosterbaan, C.W.M. van der Geld, A way to reduce pressure drop in once-through micro-evaporators, Exp. Heat Transfer, Vol. 27, DOI 10.1080/ 08916152. 2013.849183, pp. 1-11, 2014.

7. S.G. Kandlikar, "Fundamental issues related to flow boiling in minichannels and microchannels." Exp. Thermal and Fluid Science, Vol 26 (2), pp. 389-407, 2002.

8. A. Pecenko, L.T.M. van Deurzen, J.G.M. Kuerten, C.W.M. van der Geld, Non-isothermal two-phase flow with diffuse-interface model, Int. J. Multiphase Flow, Vol. 37 (2), pp. 149-165, 2011.

9. C.H.M. Baltis \& C.W.M. van der Geld, Heat transport between nucleation sites in flow boiling, submitted to Int.
J. of Heat and Mass Transfer, 2014.

10. C.H.M. Baltis \& C.W.M. van der Geld, Heat transfer mechanisms of a vapor bubble growing at a wall in saturated upward flow, submitted to J. Fluid Mech., 2014.

11 A.K. Chesters, An analytical solution for the profile and volume of a small drop or bubble symmetrical about a vertical axis, J. Fluid Mech., Vol. 81 (4), pp. 609-624, 1977.

12. C.W.M. van der Geld, C. Colin, Q.I.E. Segers, V.H. Pereira da Rosa, \& H.N. Yoshikawa, Forces on a boiling bubble in a developing boundary layer, in microgravity with g-jitter and in terrestrial conditions, Physics of Fluids, 24 (8), 082104-1/29, 2012.

13 J. Magnaudet \& D. Legendre, The viscous drag force on a spherical bubble with a time-dependent radius, Phys. of Fluids, 10, no. 3, page 550, doi:10.1063/1.869582, 1998. 